hep-th/0301035

UT-03-01

\title{
Thermal Partition Function of Superstring on Compactified PP-Wave
}

\author{
Yuji Sugawara \\ sugawara@hep-th.phys.s.u-tokyo.ac.jp \\ Department of Physics, Faculty of Science, \\ University of Tokyo \\ 7-3-1 Hongo, Bunkyo-ku, Tokyo 113-0033, Japan
}

\begin{abstract}
We study the thermal partition function of superstring on the pp-wave background with the circle compactification along a transverse direction. We calculate it in the two ways: the operator formalism and the path-integral calculation. The former gives the finite result with no subtlety of the Wick rotation, which only contains the contributions of physical states. On the other hand, the latter yields the manifestly modular invariant expression, even though we only have the winding modes along the transverse circle (no Kaluza-Klein excitations). We also check the equivalence of these two analyses. The DLCQ approach makes the path-integration quite easy. Remarkably, we find that the contributions from the transverse winding sectors disappear in the non-DLCQ limit, while they indeed contribute in the DLCQ model, depending non-trivially on the longitudinal quantum numbers.
\end{abstract}




\section{Introduction}

The string theories on the supersymmetric pp-wave backgrounds [1] have been gathering much attentions after the works [2], as new classes of exactly soluble superstring vacua [3] and as a powerful tool to analyze the AdS/CFT correspondence beyond the supergravity approximation [4].

In this article we study the thermal partition function (or the free energy in space-time theory) $[5,6,7]$ of the superstring theory on the pp-wave background compactified on a spacelike circle presented in [8]. The recent related papers analyzing the one-loop amplitudes in the strings on pp-waves are given in $[9,10,11,12,13,14,15,16,17,18,19,20]$. While the one-loop partition functions are comparably easy to analyze even in the pp-wave backgrounds, we still have several non-trivial points:

1. We need clarify the building blocks possessing good modular properties to describe the massive world-sheet theory for the transverse sector.

2. We need integrate out suitably the longitudinal degrees of freedom that are not decoupled in the relevant backgrounds.

3. It is known [8] that the world-sheet Hamiltonian in the light-cone gauge only includes the winding modes and includes no Kaluza-Klein (KK) momenta along the compactified circle. It seems quite puzzling how we can achieve modular invariant amplitudes in this situation.

The first and second points are common features in general pp-wave backgrounds with RRflux. As for the first one, only the non-trivial point is how we should evaluate the regularized zero-point energies (or the normal order constants) compatible with the good modular transformation properties. This problem has been nicely solved in [10,11]. The wanted zero-point energies are given as the Casimir energies which are defined by subtracting the divergence of "bulk" energies not sensitive to the boundary conditions. We summarize the formulas of relevant building blocks, which we call "massive theta functions" in this paper, and the zero-point energies in appendix.

The second point is much more non-trivial. In the path-integral approach we have to integrate out the longitudinal zero-modes in order to obtain modular invariant amplitudes. For the familiar string vacua with the flat space-time, the longitudinal momenta are completely decoupled and we can obtain finite results by simply taking the Wick rotation in space-time. However, in the present case of pp-wave, the naive Wick rotation leads to a difficulty of 
complex mass parameter in the light-cone gauge action. It seems quite subtle whether such models are really meaningful.

To avoid this difficulty we shall consider the thermal model as in $[13,14,15,20]$. To be more precise, we first adopt the DLCQ approach [21] and later take the decompactification limit, following our previous study [15]. In our approach the mass parameter can be always treated as a real parameter. However, as the cost we must pay for it, the longitudinal instanton actions, which capture the contributions from topological sectors, include pure imaginary terms ${ }^{1}$. Although our approach seems to have the similar difficulty of the complex worldsheet action, we can justify the result by comparing with the operator calculation, which only includes the contributions from the physical states and is a manifestly meaningful quantity.

The third point is a characteristic feature of the compactified pp-waves, and is actually the main motivation by which we study this background. This problem will be resolved in this article. As a remarkable feature, we will show that the winding modes along the transverse circle are decoupled in the non-DLCQ model, while the non-trivial contributions from them are found in the DLCQ model.

This paper is organized as follows: We first calculate the free energy by means of the operator formalism, after making a brief review on the compactified pp-wave [8]. We next derive the modular invariant expression of it based on the path-integral approach along the same line as in [15], and finally make some discussions.

\section{Operator Calculation of Space-time Free Energy in Superstring on the Compactified PP-Wave}

We start by making a brief review on the type IIB superstring theory on the maximally supersymmetric pp-wave compactified around a space-like circle [8] to mainly prepare our notations. (A good review is also found in the recent paper [23].) Suppose a compactification along a circle in the $X^{1}-X^{2}$ plane in the space-like directions. According to [8], we introduce the following new coordinates which simplify a Killing vector on the pp-wave background $(\mu$ is the strength of 5 -form RR-flux);

$$
X^{+}=Z^{+}, \quad X^{-}=Z^{-}-\mu Z^{1} Z^{2},
$$

\footnotetext{
${ }^{1} \mathrm{We}$ would like to emphasize that this is not a peculiarity of the pp-wave. This phenomenon appears already in the thermal model of flat DLCQ string, and is justified by comparing with the operator calculation $[22]$.
} 


$$
\left(\begin{array}{c}
X^{1} \\
X^{2}
\end{array}\right)=\left(\begin{array}{cc}
\cos \left(\mu X^{+}\right) & -\sin \left(\mu X^{+}\right) \\
\sin \left(\mu X^{+}\right) & \cos \left(\mu X^{+}\right)
\end{array}\right)\left(\begin{array}{c}
Z^{1} \\
Z^{2}
\end{array}\right)
$$

and the other coordinates $X^{I}(I=3, \ldots, 8)$ remain unchanged. Because the relevant Killing vector is now written as $V=\partial / \partial Z^{1}$, we can take an $S^{1}$-compactification

$$
Z^{1} \sim Z^{1}+2 \pi R_{T}
$$

which is known to give a supersymmetric sting vacuum with 24 Killing spinors [8]. Under the light-cone gauge

$$
Z^{+}=\alpha^{\prime} p^{+} \tau, \quad \Gamma^{+} \theta=\Gamma^{+} \tilde{\theta}=0
$$

the Green-Schwarz action is written as

$$
\begin{aligned}
S= & S_{B}+S_{F} \\
S_{B}= & \frac{1}{4 \pi \alpha^{\prime}} \int d^{2} \sigma\left[\partial_{+} Z^{1} \partial_{-} Z^{1}+\partial_{+} Z^{2} \partial_{-} Z^{2}+\partial_{+} X^{I} \partial_{-} X^{I}\right. \\
& \left.\quad-m^{2}\left(X^{I}\right)^{2}-4 m Z^{2} \partial_{\tau} Z^{1}\right], \\
S_{F}= & \frac{i}{2 \pi} \int d^{2} \sigma\left[S\left(\partial_{+}-\frac{m}{2} \gamma^{1} \bar{\gamma}^{2}\right) S+\tilde{S}\left(\partial_{-}-\frac{m}{2} \gamma^{1} \bar{\gamma}^{2}\right) \tilde{S}-2 m S \Pi \tilde{S}\right],
\end{aligned}
$$

where $m=\mu \alpha^{\prime} p^{+}$and $\partial_{ \pm} \equiv \partial_{\tau} \pm \partial_{\sigma}$ in our convention. The $8 \times 8$ matrices $\gamma^{i}, \bar{\gamma}^{i}(i=$ $1, \ldots, 8)$ are defined so that the chiral representation of $S O(8)$ gamma matrices is given by $\left(\begin{array}{cc}0 & \gamma^{i} \\ \bar{\gamma}^{i} & 0\end{array}\right)$, and satisfy

$$
\gamma^{i} \bar{\gamma}^{j}+\gamma^{j} \bar{\gamma}^{i}=2 \delta^{i j}, \quad \bar{\gamma}^{i} \gamma^{j}+\bar{\gamma}^{j} \gamma^{i}=2 \delta^{i j}, \quad\left(\gamma^{i}\right)^{T}=\bar{\gamma}^{i}
$$

We also set $\Pi=\gamma^{1} \bar{\gamma}^{2} \gamma^{3} \bar{\gamma}^{4}$. We note that the the coordinate system (2.1) reduces to the rotated frame after taking the light-cone gauge. The absence of the harmonic potential (mass term) and the existence of interaction with a constant magnetic field (or the Coriolis force) for $Z^{1}, Z^{2}$ is originating from this fact.

Combining $Z^{1}, Z^{2}$ to a complex boson $Z \equiv Z^{1}+i Z^{2}$, the equations of motion are written as

$$
\begin{aligned}
& \partial_{+} \partial_{-} Z+2 i m \partial_{\tau} Z=0, \\
& \partial_{+} \partial_{-} X^{I}+m^{2} X^{I}=0, \quad(I=3, \ldots, 8) \\
& \left(\partial_{+}-\frac{m}{2} \gamma^{1} \bar{\gamma}^{2}\right) S-m \Pi \tilde{S}=0, \\
& \left(\partial_{-}-\frac{m}{2} \gamma^{1} \bar{\gamma}^{2}\right) \tilde{S}+m \Pi S=0 .
\end{aligned}
$$


It is convenient to solve the equation for $Z$ in the following form;

$$
Z=e^{-i m \tau} Y+W R_{T} \sigma+\left(Z_{0}^{1}+i Z_{0}^{2}\right)
$$

where $Y$ is the standard massive complex boson satisfying $\partial_{+} \partial_{-} Y+m^{2} Y=0 . W(\in \mathbf{Z})$ is the winding number for the circle along $Z^{1}$ and $Z_{0} \equiv Z_{0}^{1}+i Z_{0}^{2}$ (= const.) is the center of mass coordinate. We remark that the zero-mode part of the solution (2.8) does not include the KK momentum term $\propto \tau$, which is not compatible with the equations of motion (2.7). Similarly, for the fermionic coordinates, it is convenient to rewrite as

$$
S=e^{\frac{m}{2} \gamma^{1} \bar{\gamma}^{2} \tau} \Sigma, \quad \tilde{S}=e^{\frac{m}{2} \gamma^{1} \bar{\gamma}^{2} \tau} \tilde{\Sigma},
$$

where $\left(\Sigma^{a}, \tilde{\Sigma}^{a}\right)$ satisfy the usual Dirac equation with mass $m$;

$$
\begin{aligned}
& \partial_{+} \Sigma-m \Pi \tilde{\Sigma}=0, \\
& \partial_{-} \tilde{\Sigma}+m \Pi \Sigma=0 .
\end{aligned}
$$

The canonically conjugate momenta are given as

$$
\begin{aligned}
\Pi_{I} & =\frac{1}{2 \pi \alpha^{\prime}} \partial_{\tau} X^{I}, \quad \Pi_{1}=\frac{1}{2 \pi \alpha^{\prime}}\left(\partial_{\tau} Z^{1}-2 m Z^{2}\right), \quad \Pi_{2}=\frac{1}{2 \pi \alpha^{\prime}} \partial_{\tau} Z^{2}, \\
\lambda^{a} & =\frac{i}{2 \pi} S^{a}, \quad \tilde{\lambda}^{a}=\frac{i}{2 \pi} \tilde{S}^{a} .
\end{aligned}
$$

Since $\Pi_{1}$ is canonically conjugate to the compactified coordinate $Z^{1}$, it must be quantized as

$$
\int_{0}^{2 \pi} d \sigma \Pi_{1}=\frac{k}{R_{T}}, \quad(k \in \mathbf{Z}) .
$$

We thus find the quantization of zero-mode coordinate $Z_{0}^{2}$, since $\int_{0}^{2 \pi} d \sigma \partial_{\tau} Z^{1}=0$ holds as we mentioned above. This phenomenon is the well-known one, giving rise to the degeneracy of the "Landau levels";

$$
\mathcal{N}=\frac{2 \pi R_{T}^{\prime}}{\left(\alpha^{\prime} / 2 m R_{T}\right)}=\frac{1}{\alpha^{\prime}} 4 \pi m R_{T} R_{T}^{\prime}
$$

where we set the volume along the $Z^{2}$-direction $2 \pi R_{T}^{\prime}$, which should be decompactified after the calculation.

The canonical quantization is defined in the standard manner. The easiest way to do so is to introduce the mode expansions with respect to $Y, Y^{*}, \Sigma^{a}, \tilde{\Sigma}^{a}$ (and $X^{I}$, of course), since they satisfy the same equations of motion as in [3]. The Virasoro constraints lead us to the 
following world-sheet Hamiltonian

$$
\begin{aligned}
H\left(\equiv-\alpha^{\prime} p^{+} p^{-}\right)= & \int d \sigma\left(\Pi_{r} \partial_{\tau} Z^{r}+\Pi_{I} \partial_{\tau} X^{I}+\lambda_{a} \partial_{\tau} S^{a}+\tilde{\lambda}_{a} \partial_{\tau} \tilde{S}^{a}-L\right) \\
= & \frac{W^{2} R_{T}^{2}}{2 \alpha^{\prime}}+2 m N_{0}^{(+)}+\sum_{n \neq 0}\left[\left(\omega_{n}+m\right) N_{n}^{(+)}+\left(\omega_{n}-m\right) N_{n}^{(-)}\right]+\sum_{I=3}^{8} \sum_{n \in \mathbf{Z}} \omega_{n} N_{n}^{I} \\
& \quad+\sum_{n \in \mathbf{Z}}\left[\left(\omega_{n}+\frac{m}{2}\right) N_{F, n}^{(+)}+\left(\omega_{n}-\frac{m}{2}\right) N_{F, n}^{(-)}\right]
\end{aligned}
$$

In this expression we set $\omega_{n}=\sqrt{n^{2}+m^{2}} . N_{n}^{I}$ are the mode counting operators for $X^{I}$, and $N_{n}^{(+)}\left(N_{n}^{(-)}\right)$is the one associated to the positive (negative) frequency modes of $Y$ and the negative (positive) frequency modes of $Y^{*}$. The fermionic mode counting operators $N_{F, n}^{( \pm)}$are defined as follows: Let $S=S^{(+)}+S^{(-)}, \tilde{S}=\tilde{S}^{(+)}+\tilde{S}^{(-)}$be the decomposition according to the eigen-value of $i \gamma^{1} \bar{\gamma}^{2}(= \pm 1) . N_{F, n}^{(+)}\left(N_{F, n}^{(-)}\right)$is the one associated to the positive (negative) frequency modes of $S^{(+)}, \tilde{S}^{(+)}$and the negative (positive) frequency modes of $S^{(-)}, \tilde{S}^{(-)}$. We have no normal order constant here thanks to the SUSY cancellation, since both the bosonic and fermionic coordinates obey the periodic boundary conditions. We will later face the situations in which this cancellation fails due to the twisted boundary conditions in the calculation of thermal amplitudes. In those cases we need the non-trivial formula of regularized zero-point energies (A.2) given in $[10,11]$ (see also $[14,15,16]$ ). It is obvious that the energies of each bosonic and fermionic oscillators in the Hamiltonian (2.14) are not balanced. This fact implies that all of the 24 Killing spinors are "time-dependent", corresponding to the supercharges that do not commute with the Hamiltonian.

The Virasoro condition also provides the level matching condition in the standard manner;

$$
P_{\text {osc }}-k W=0,
$$

where $P_{\text {osc }}$ means the oscillator part of the world-sheet momentum operator defined as

$$
P_{\mathrm{osc}}=\sum_{n \in \mathbf{Z}} n\left[N_{n}^{(+)}+N_{n}^{(-)}+\sum_{I} N_{n}^{I}+N_{F, n}^{(+)}+N_{F, n}^{(-)}\right] .
$$

Notice that the canonical momentum $k \propto \int d \sigma \Pi_{1}$ is absent in the Hamiltonian (2.14) as is expected, but the level-matching condition depends on it.

For later convenience, we shall take the DLCQ compactification [21] from now on;

$$
Z^{-} \sim Z^{-}+2 \pi R_{-} .
$$

This is really well-defined since $\partial / \partial Z^{-}$is still a covariantly constant Killing vector in the rotated coordinates (2.1). Under (2.17), the light-cone momentum is quantized as

$$
p^{+}=\frac{p}{R_{-}}, \quad\left(p \in \mathbf{Z}_{>0}\right),
$$


and the level-matching condition (2.15) is deformed as

$$
P_{\mathrm{osc}}-k W \in p \mathbf{Z}
$$

Now, we are in the position to calculate the free energy (with vanishing chemical potential) in the thermal ensemble of free strings on the relevant pp-wave backgrounds. Let $\beta$ be the inverse temperature, then the free energy should be written as

$$
\begin{aligned}
F(\beta) & =\frac{1}{\beta} \operatorname{Tr}\left[(-1)^{\mathbf{F}} \ln \left(1-(-1)^{\mathbf{F}} e^{-\beta p^{0}}\right)\right] \\
& \equiv-\sum_{n=1}^{\infty} \frac{1}{\beta n} \operatorname{Tr}\left[(-1)^{(n+1) \mathbf{F}} e^{-\beta n p^{0}}\right]
\end{aligned}
$$

where $\mathbf{F}$ denotes the space-time fermion number $(\bmod 2)$ and $p^{0} \equiv \frac{1}{\sqrt{2}}\left(p^{+}-p^{-}\right)$is the spacetime energy operator. The trace should be taken over the single particle physical Hilbert space on which the on-shell condition and the level matching condition are imposed. The following calculation is almost parallel to that of [15]. In the present problem, however, we must care about the degeneracy of Landau levels. We thus calculate the free energy, dividing it by the degeneracy $\mathcal{N}$ given in (2.13), and then take the $R_{T}^{\prime} \rightarrow \infty$ limit.

The on-shell condition is written as

$$
p^{0}=\frac{1}{\sqrt{2}}\left(p^{+}-p^{-}\right)=\frac{1}{\sqrt{2}}\left(\frac{p}{R_{-}}+\frac{R_{-}}{\alpha^{\prime} p} H\right) .
$$

Imposing the level matching condition (2.19) is slightly a non-trivial task. It is achieved by inserting the following projection operator into the trace

$$
\frac{1}{p} \sum_{q \in \mathbf{Z}_{p}} e^{2 \pi i \frac{q}{p}\left(P_{\mathrm{osc}}-k W\right)} .
$$

We so obtain

$$
\begin{aligned}
F(\beta)=- & \lim _{R_{T}^{\prime} \rightarrow \infty} \frac{1}{\mathcal{N}} \sum_{n=1}^{\infty} \sum_{p=1}^{\infty} \sum_{q \in \mathbf{Z}_{p}} \sum_{W \in \mathbf{Z}} \sum_{k=0}^{\mathcal{N}} \frac{1}{\beta n p} e^{-\frac{\beta n p}{\sqrt{2} R_{-}}-\frac{\beta n R_{-}}{\sqrt{2} \alpha^{\prime} p} \cdot \frac{R_{T}^{2} W^{2}}{2 \alpha^{\prime}}} \cdot e^{-2 \pi i \frac{q}{p} k W} \\
& \times \operatorname{Tr}\left[(-1)^{(n+1) \mathbf{F}} e^{-\beta n \frac{R}{\sqrt{2} p \alpha^{\prime}} H_{\text {osc }}+2 \pi i \frac{q}{p} P_{\text {osc }}}\right]
\end{aligned}
$$

where $H_{\text {osc }}$ denotes the oscillator part of Hamiltonian (2.14). The trace is taken over the Fock space associated to the light-cone momentum $p^{+}=p / R_{-}$. The summation of $k$ is readily carried out under the large $R_{T}^{\prime}$-limit (large $\mathcal{N}$-limit) as

$$
\lim _{R_{T}^{\prime} \rightarrow \infty} \frac{1}{\mathcal{N}} \sum_{k=0}^{\mathcal{N}} e^{-2 \pi i \frac{q}{p} k W}=\sum_{k^{\prime} \in \mathbf{Z}} \hat{\delta}\left(q W-p k^{\prime}\right),
$$


where we set

$$
\hat{\delta}(n)=\left\{\begin{array}{ll}
1 & (n=0) \\
0 & (n \neq 0)
\end{array} \quad(n \in \mathbf{Z})\right.
$$

Let us introduce $d \stackrel{\text { def }}{=}|\mathbf{G C D}(p, q)|$, and write $p=\bar{p} d, q=\bar{q} d$. Since $\bar{p}$ and $\bar{q}$ are relatively prime, the "discrete delta function" (2.25) imposes that $W$ must be written as the form

$$
W=\bar{p} \ell, \quad(\ell \in \mathbf{Z})
$$

It is also convenient to introduce the "modulus parameter" $\tau \equiv \frac{q+i n \nu}{p}$ with the constant $\nu \equiv \frac{\sqrt{2} \beta R_{-}}{4 \pi \alpha^{\prime}}$. The trace of oscillator part is now written as $\operatorname{Tr}\left[(-1)^{(n+1) \mathbf{F}} e^{-2 \pi \tau_{2} H_{\mathrm{osc}}+2 \pi i \tau_{1} P_{\mathrm{osc}}}\right]$. This is easily evaluated from the explicit form of Hamiltonian (2.14) and expressed concisely in terms of the massive theta functions defined in (A.1). The temporal boundary condition of fermionic coordinates should be periodic for even $n$ and anti-periodic for odd $n$ because of the insertion of $(-1)^{(n+1) \mathbf{F}}$. Combining all the things, we finally obtain

$$
\begin{gathered}
F(\beta)=-2 \pi R_{T} \sum_{\epsilon=0,1} \sum_{n \in 2 \mathbf{Z}+\epsilon, n>0} \sum_{p, q} \sum_{W \in(p / d) \mathbf{Z}} \frac{1}{\beta n p} e^{-\frac{\beta^{2} n^{2}}{4 \pi \alpha^{\prime} \tau_{2}}\left(1+\frac{R_{-}^{2} R_{T}^{2} W^{2}}{2 p^{2} \alpha^{\prime 2}}\right)} \\
\times \frac{\Theta_{\left(0, i \frac{\hat{\mu} \nu n}{2}+\frac{\epsilon}{2}\right)}(\tau, \bar{\tau} ; \hat{\mu} p)^{4}}{\Theta_{(0,0)}(\tau, \bar{\tau} ; \hat{\mu} p)^{3} \cdot \Theta_{(0, i \hat{\mu} \nu n)}(\tau, \bar{\tau} ; \hat{\mu} p)},
\end{gathered}
$$

where the integers $n, p(>0), q$ run over the range such that $\tau \in \mathcal{S}$ with the definition

$$
\mathcal{S} \stackrel{\text { def }}{=}\left\{\tau \in \mathbf{C} ; \tau_{2}>0,\left|\tau_{1}\right| \leq \frac{1}{2}\right\} .
$$

It is interesting that the winding modes $W$ along the space-like circle is not independent of the longitudinal quantum numbers $p, q$. This feature is in a sharp contrast with the flat backgrounds.

Let us finally discuss the decompactification limit $R_{-} \rightarrow \infty$. The desired free energy should be defined as $\lim _{R_{-} \rightarrow \infty} \frac{F(\beta)}{\sqrt{2} \pi R_{-}}$, in which the summation of $p$ is replaced with the integration

$$
\frac{1}{R_{-}} \sum_{p} f\left(p / R_{-}\right) \longrightarrow \int_{0}^{\infty} d p^{+} f\left(p^{+}\right)
$$

To consider this limit we first note that the winding modes $W \neq 0$ are trivially decoupled, unless $d \equiv|\mathbf{G C D}(p, q)|$ is the value of the same order of $R_{-}$. In the cases when $d \sim O\left(R_{-}\right)$ 
holds and $W \neq 0$, however, the discrete sum $\frac{1}{p} \sum_{q} *$ is still negligible because of the small factor $1 / p \sim O\left(1 / R_{-}\right)$. We thus conclude that only the no winding sector $W=0$ survives and the summation of $q$ reduces to an integral

$$
\frac{1}{p} \sum_{q} f(q / p) \longrightarrow \int_{-1 / 2}^{1 / 2} d \tau_{1} f\left(\tau_{1}\right) \text {. }
$$

In this way, we have achieved the wanted decompactification limit;

$$
\begin{aligned}
\lim _{R_{-} \rightarrow \infty} \frac{F(\beta)}{\sqrt{2} \pi R_{-}}=-2 \pi R_{T} \sum_{\epsilon=0,1} & \sum_{n \in 2 \mathbf{Z}+\epsilon, n>0} \frac{1}{\beta n} \int_{0}^{\infty} \frac{d p^{+}}{\sqrt{2} \pi} \int_{-1 / 2}^{1 / 2} d \tau_{1} e^{-\frac{\beta n p}{\sqrt{2}}} \\
& \times \frac{\Theta_{\left(0, i \frac{\sqrt{2} \mu \beta}{8 \pi} n+\frac{\epsilon}{2}\right)}\left(\tau, \bar{\tau} ; \mu \alpha^{\prime} p^{+}\right)^{4}}{\Theta_{(0,0)}\left(\tau, \bar{\tau} ; \mu \alpha^{\prime} p^{+}\right)^{3} \cdot \Theta_{\left(0, i \frac{\sqrt{2} \mu \beta}{4 \pi} n\right)}\left(\tau, \bar{\tau} ; \mu \alpha^{\prime} p^{+}\right)},
\end{aligned}
$$

where we set $\tau=\tau_{1}+i \frac{\sqrt{2} \beta n}{4 \pi \alpha^{\prime} p^{+}}$. It is also not difficult to derive (2.31) directly from the non-DLCQ model. The light-cone momentum $p^{+}$is now continuous. The level matching condition is given by (2.15) instead of (2.19), and hence the projection operator (2.22) should be replaced with

$$
\int_{-1 / 2}^{1 / 2} d \tau_{1} e^{2 \pi i \tau_{1}\left(P_{\mathrm{osc}}-k W\right)}
$$

(2.24) is further replaced with

$$
\lim _{R_{T}^{\prime} \rightarrow \infty} \frac{1}{\mathcal{N}} \sum_{k=0}^{\mathcal{N}} e^{-2 \pi i \tau_{1} k W}= \begin{cases}1 & (W=0) \\ \lim _{R_{T}^{\prime} \rightarrow \infty} \frac{1}{\mathcal{N}} \sum_{k^{\prime} \in \mathbf{Z}} \delta\left(W \tau_{1}-k^{\prime}\right) & (W \neq 0)\end{cases}
$$

Therefore, we again find that the $W \neq 0$ sectors are decoupled, and obtain the same result $(2.31)$.

\section{Path-Integral Calculation of Thermal Partition Func- tion in Superstring on the Compactified PP-Wave}

Next we perform the path-integral calculation of the toroidal partition function with the thermal compactification. The wanted partition function $Z_{\text {torus }}(\beta)$ will have a manifestly modular invariant form and should be equated with the free energy considered above by the next simple relation;

$$
Z_{\text {torus }}(\beta)=-\beta F(\beta)
$$


We shall first deal with the DLCQ model, and will later consider the decompactification limit. The calculation is again almost parallel to that presented in [15] (see also [22]). We need the Wick rotation in both of the world-sheet and space-time. Although the Wick rotated pp-wave backgrounds have a difficulty of complex mass parameter as we already mentioned, in our approach the mass parameter $m$ can be always treated as a real parameter. As the cost we must pay for it, the longitudinal string coordinates become complex, leading to a complex instanton action. Nevertheless, the final result will turn out to be a real function and to be justified by confirming the relation (3.1).

We define the Wick rotated world-sheet coordinates as

$$
\sigma_{1}=\sigma, \quad \sigma_{2}=i \tau
$$

In the Wick rotated space-time $Z^{ \pm} \equiv \frac{1}{\sqrt{2}}\left(Z^{9} \pm i Z_{E}^{0}\right)$, the DLCQ string theory $\left(Z^{-} \sim Z^{-}+\right.$ $\left.2 \pi R_{-}\right)$is described by the complex identification

$$
Z_{E}^{0} \sim Z_{E}^{0}+\sqrt{2} \pi R_{-} i, \quad Z^{9} \sim Z^{9}+\sqrt{2} \pi R_{-},
$$

and the thermal compactification is defined as

$$
Z_{E}^{0} \sim Z_{E}^{0}+\beta
$$

where $\beta$ denotes the inverse temperature.

The longitudinal coordinates have various topological sectors;

$$
\begin{aligned}
Z^{+}\left(\sigma_{1}+2 \pi, \sigma_{2}\right) & =Z^{+}\left(\sigma_{1}, \sigma_{2}\right)+\frac{i \beta}{\sqrt{2}} w, \\
Z^{+}\left(\sigma_{1}+2 \pi \tau_{1}, \sigma_{2}+2 \pi \tau_{2}\right)= & Z^{+}\left(\sigma_{1}, \sigma_{2}\right)+\frac{i \beta}{\sqrt{2}} n, \\
Z^{-}\left(\sigma_{1}+2 \pi, \sigma_{2}\right)= & Z^{-}\left(\sigma_{1}, \sigma_{2}\right)-\frac{i \beta}{\sqrt{2}} w+2 \pi R_{-} r, \\
Z^{-}\left(\sigma_{1}+2 \pi \tau_{1}, \sigma_{2}+2 \pi \tau_{2}\right)= & Z^{-}\left(\sigma_{1}, \sigma_{2}\right)-\frac{i \beta}{\sqrt{2}} n+2 \pi R_{-} s, \\
& (w, n, r, s \in \mathbf{Z}) .
\end{aligned}
$$

Let $Z_{w, n, r, s}^{+}, Z_{w, n, r, s}^{-}$be the instanton solution obeying the boundary conditions (3.5). Even though the light-cone gauge is not compatible with (3.5), we can take the "instanton gauge" $Z^{+}=Z_{w, n, r, s}^{+}$, which makes the world-sheet action quadratic as in [15]. In fact, consider the rotation of the world-sheet coordinates as

$$
\left(\begin{array}{c}
\sigma_{1}^{\prime} \\
\sigma_{2}^{\prime}
\end{array}\right)=\left(\begin{array}{cc}
\cos \theta_{w, n} & -\sin \theta_{w, n} \\
\sin \theta_{w, n} & \cos \theta_{w, n}
\end{array}\right)\left(\begin{array}{c}
\sigma_{1} \\
\sigma_{2}
\end{array}\right)
$$


with

$$
\cos \theta_{w, n}=\frac{w \tau_{1}-n}{|w \tau-n|}, \quad \sin \theta_{w, n}=-\frac{w \tau_{2}}{|w \tau-n|} .
$$

Then, $Z_{w, n, r, s}^{+}$behaves in this new coordinate as

$$
Z_{w, n, r, s}^{+}=-i \frac{\sqrt{2} \beta}{4 \pi \tau_{2}}|w \tau-n| \sigma_{2}^{\prime}
$$

As a result, we obtain the equation of motion of the same form as (2.7) (on the Euclidean world-sheet);

$$
\left(\partial_{\sigma_{1}^{\prime}}^{2}+\partial_{\sigma_{2}^{\prime}}^{2}\right) Z+2 m \partial_{\sigma_{2}^{\prime}} Z=0
$$

but with the non-trivial mass parameter

$$
m=\mu \frac{\sqrt{2} \beta}{4 \pi \tau_{2}}|w \tau-n| \equiv \hat{\mu} \frac{\nu}{\tau_{2}}|w \tau-n| .
$$

This again leads to

$$
\left(\partial_{\sigma_{1}^{\prime}}^{2}+\partial_{\sigma_{2}^{\prime}}^{2}\right) Y-m^{2} Y=0
$$

under the transformation of string coordinates

$$
Z=e^{-m \sigma_{2}^{\prime}} Y+a R_{T} \sigma_{1}^{\prime}+Z_{0}
$$

where $a$ is a real number (not necessarily an integer) which will be determined by the boundary conditions of $Z$. Since (3.11) has the manifest rotational symmetry, we can move back to the original coordinates $\sigma_{1}, \sigma_{2}$ which is independent of the modulus $\tau$ without changing the form of equation;

$$
\left(\partial_{\sigma_{1}}^{2}+\partial_{\sigma_{2}}^{2}\right) Y-m^{2} Y=0
$$

This fact makes things quite easy. We can calculate the one-loop amplitude as the determinant of standard Kaluza-Klein operator, resulting the massive theta functions (A.1). However, $Y$ obeys the non-trivial boundary conditions as the function of $\sigma_{1}, \sigma_{2}$. We must carefully evaluate them from the boundary conditions of $Z$;

$$
\begin{aligned}
& Z\left(\sigma_{1}+2 \pi, \sigma_{2}\right)-Z\left(\sigma_{1}, \sigma_{2}\right) \in 2 \pi R_{T} \mathbf{Z}, \\
& Z\left(\sigma_{1}+2 \pi \tau_{1}, \sigma_{2}+2 \pi \tau_{2}\right)-Z\left(\sigma_{1}, \sigma_{2}\right) \in 2 \pi R_{T} \mathbf{Z} .
\end{aligned}
$$

Based on (3.6), (3.7) and (3.12) we first find the constraints on $a$;

$$
\text { (*) : } \frac{w \tau_{1}-n}{|w \tau-n|} a \in \mathbf{Z}, \quad \frac{w|\tau|^{2}-n \tau_{1}}{|w \tau-n|} a \in \mathbf{Z} .
$$


These constraints are quite non-trivial to solve, and probably we could not have the solutions everywhere on the moduli space of torus. However, it will later turn out that we can always solve it for the DLCQ model which effectively has the discretized moduli space. Secondly, the periodicity of the oscillator part of $Z$ leads to

$$
\begin{aligned}
Y\left(\sigma_{1}+2 \pi, \sigma_{2}\right) & =e^{-2 \pi \hat{\mu} \nu w} Y\left(\sigma_{1}, \sigma_{2}\right) \\
Y\left(\sigma_{1}+2 \pi \tau_{1}, \sigma_{2}+2 \pi \tau_{2}\right) & =e^{-2 \pi \hat{\mu} \nu n} Y\left(\sigma_{1}, \sigma_{2}\right) .
\end{aligned}
$$

Similar arguments work also for the fermionic coordinates. We finally achieve the GS fermions $\Sigma, \tilde{\Sigma}$ that satisfy the Dirac equation with the mass (3.10)

$$
\begin{aligned}
& \left(\partial_{\sigma_{1}}+i \partial_{\sigma_{2}}\right) \Sigma-m \Pi \tilde{\Sigma}=0 \\
& \left(\partial_{\sigma_{1}}-i \partial_{\sigma_{2}}\right) \tilde{\Sigma}-m \Pi \Sigma=0
\end{aligned}
$$

and obey the boundary conditions

$$
\begin{aligned}
\Sigma\left(\sigma_{1}+2 \pi, \sigma_{2}\right) & =(-1)^{w} e^{-i \pi \hat{\mu} \nu w \gamma^{1} \bar{\gamma}^{2}} \Sigma\left(\sigma_{1}, \sigma_{2}\right), \\
\tilde{\Sigma}\left(\sigma_{1}+2 \pi, \sigma_{2}\right) & =(-1)^{w} e^{-i \pi \hat{\mu} \nu w \gamma^{1} \bar{\gamma}^{2}} \tilde{\Sigma}\left(\sigma_{1}, \sigma_{2}\right), \\
\Sigma\left(\sigma_{1}+2 \pi \tau_{1}, \sigma_{2}+2 \pi \tau_{2}\right) & =(-1)^{n} e^{-i \pi \hat{\mu} \nu n \gamma^{1} \bar{\gamma}^{2}} \Sigma\left(\sigma_{1}, \sigma_{2}\right), \\
\tilde{\Sigma}\left(\sigma_{1}+2 \pi \tau_{1}, \sigma_{2}+2 \pi \tau_{2}\right) & =(-1)^{n} e^{-i \pi \hat{\mu} \nu n \gamma^{1} \bar{\gamma}^{2}} \tilde{\Sigma}\left(\sigma_{1}, \sigma_{2}\right) .
\end{aligned}
$$

Here the extra phase factors $(-1)^{w},(-1)^{n}$ are due to the thermal boundary conditions for world-sheet fermions [7].

The contributions from the zero-modes are described by the instanton actions, being summed over possible topological sectors. For the longitudinal modes we obtain

$$
\frac{V_{\text {l.c. }}}{4 \pi^{2} \alpha^{\prime} \tau_{2}} \times \sum_{w, n, r, s} e^{-S_{\mathrm{inst}}(w, n, r, s)}=\frac{\nu}{\tau_{2}} \sum_{w, n, r, s} e^{-S_{\mathrm{inst}}(w, n, r, s)}
$$

where the instanton action is evaluated as

$$
S_{\text {inst }}(w, n, r, s)=\frac{\beta^{2}|w \tau-n|^{2}}{4 \pi \alpha^{\prime} \tau_{2}}+2 \pi i \frac{\nu}{\tau_{2}}\left\{|\tau|^{2} w r-\tau_{1}(w s+n r)+n s\right\} .
$$

$V_{\text {l.c. }}=\sqrt{2} \pi R_{-} \beta$ denotes the volume of the longitudinal directions and we again set $\nu \equiv$ $\frac{\sqrt{2} \beta R_{-}}{4 \pi \alpha^{\prime}}$. We here define the amplitude by subtracting the $w=n=0$ sector following the arguments given in [22], which could induce a trivial divergent sum of the remaining winding numbers $r, s$. This term corresponds to the total vacuum energy under the zero temperature limit $\beta \rightarrow \infty$. We will discuss it later. 
We also obtain for the zero-modes along the space-like circle

$$
2 \pi R_{T} \sum_{a:(*)} e^{-\frac{\pi R_{T}^{2}\left|W_{1}(a) \tau-W_{2}(a)\right|^{2}}{\alpha^{\prime} \tau_{2}}},
$$

where the summation is over all the real numbers $a$ satisfying the constraints $(*)$ given in (3.15) and the winding numbers $W_{1}(a), W_{2}(a)$ are determined by the relations

$$
W_{1}(a)=\frac{w \tau_{1}-n}{|w \tau-n|} a, \quad W_{2}(a)=\frac{w|\tau|^{2}-n \tau_{1}}{|w \tau-n|} a .
$$

Taking all the things into account, we obtain the following partition function

$$
\begin{aligned}
Z_{\text {torus }}(\beta)=2 \pi R_{T} \nu \int_{\mathcal{F}} \frac{d^{2} \tau}{\tau_{2}^{2}} & \sum_{\epsilon_{i}=0,1} \sum_{\substack{w \in 2 \mathbf{Z}_{+} \epsilon_{1} \\
n \in 2 \mathbf{Z}+\epsilon_{2}}}^{\prime} \sum_{r, s} \sum_{a:(*)} e^{-S_{\mathrm{inst}}(w, n, r, s)-\frac{\pi R_{T}^{2}\left|W_{1}(a) \tau-W_{2}(a)\right|^{2}}{\alpha^{\prime} \tau_{2}}} \\
& \times \frac{\Theta_{\left(-i \frac{\hat{\mu} \nu w}{2}+\frac{\epsilon_{1}}{2}, i \frac{\hat{\mu} \nu n}{2}+\frac{\epsilon_{2}}{2}\right)}(\tau, \bar{\tau} ; m)^{4}}{\Theta_{(0,0)}(\tau, \bar{\tau} ; m)^{3} \cdot \Theta_{(-i \hat{\mu} \nu w, i \hat{\mu} \nu n)}(\tau, \bar{\tau} ; m)},
\end{aligned}
$$

where we set $m \equiv \hat{\mu} \frac{\nu}{\tau_{2}}|w \tau-n|$, and $\sum^{\prime}$ indicates the summation over $w, n$ except for $w=$ $n=0 . \mathcal{F}$ denotes the fundamental domain of moduli space

$$
\mathcal{F} \stackrel{\text { def }}{=}\left\{\tau \in \mathbf{C} ; \tau_{2}>0,|\tau|>1,\left|\tau_{1}\right| \leq \frac{1}{2}\right\}
$$

However, (3.23) is not the desired one, because the condition (*) cannot be easily solved and the windings $W_{1}(a), W_{2}(a)$ have complicated forms depending non-trivially on the modulus $\tau$. Fortunately, we can improve this point drastically in our DLCQ approach. In fact, we can explicitly carry out the summations over $r, s$, since the remaining sectors do not depend on them. (Recall our assumption $(w, n) \neq(0,0)$.) We so find the periodic delta function term;

$$
\sum_{r, s} e^{-S_{\mathrm{inst}}(w, n, r, s)}=\sum_{p, q} e^{-\frac{\beta^{2}|w \tau-n|^{2}}{4 \pi \alpha^{\prime} \tau_{2}}} \tau_{2} \delta^{(2)}((w \nu+i p) \tau-(n \nu+i q))
$$

which imposes the constraints

$$
\begin{aligned}
& w \nu \tau_{1}-p \tau_{2}=n \nu, \\
& w \nu \tau_{2}+p \tau_{1}=q .
\end{aligned}
$$

With the helps of them, the condition $(*)$ is explicitly solved;

$$
a=\frac{\ell \nu}{d \tau_{2}}|w \tau-n|, \quad\left({ }^{\forall} \ell \in \mathbf{Z}\right),
$$


and $W_{1}(a), W_{2}(a)$ are determined as

$$
W_{1}(a)=\bar{p} \ell, \quad W_{2}(a)=\bar{q} \ell
$$

where we again set $d=|\operatorname{GCD}(p, q)|, p=\bar{p} d, q=\bar{q} d$. After a short calculation using again (3.26), we finally achieve the expression as follows;

$$
\begin{aligned}
& Z_{\text {torus }}(\beta)=2 \pi R_{T} \nu \int_{\mathcal{F}} \frac{d^{2} \tau}{\tau_{2}^{2}} \sum_{\epsilon_{i}=0,1} \sum_{\substack{w \in 2 \mathbf{Z}_{+\epsilon_{1}} \\
n \in 2 \mathbf{Z}+\epsilon_{2}}}^{\prime} \sum_{W \in(p / d) \mathbf{Z}} e^{-\frac{\beta^{2}|w \tau-n|^{2}}{4 \pi \alpha^{\prime} \tau_{2}}\left(1+\frac{R_{-}^{2} R_{T}^{2} W^{2}}{2 p^{2} \alpha^{\prime 2}}\right)} \\
& \quad \times \tau_{2} \delta^{(2)}((w \nu+i p) \tau-(n \nu+i q)) \frac{\Theta_{\left(-i \frac{\hat{\mu} w \nu}{2}+\frac{1}{2} \epsilon_{1}, i \frac{\hat{\mu} n \nu}{2}+\frac{1}{2} \epsilon_{2}\right)}(\tau, \bar{\tau} ; \hat{\mu}|w \nu+i p|)^{4}}{\Theta_{(0,0)}(\tau, \bar{\tau} ; \hat{\mu}|w \nu+i p|)^{3} \cdot \Theta_{(-i \hat{\mu} w \nu, i \hat{\mu} n \nu)}(\tau, \bar{\tau} ; \hat{\mu}|w \nu+i p|)} .
\end{aligned}
$$

This is the desired partition function. The modular invariance of integrand is confirmed because of (A.3). Especially, the $S$-transformation brings about the exchanges $p \leftrightarrow-q$, $w \leftrightarrow-n$, and leaves $d \equiv|\mathbf{G C D}(p, q)|$ unchanged.

It is now straightforward to check the equivalence with the operator calculation. In fact, the modular invariance allows us to make (3.29) a simpler form by setting $w=0$ and replacing $\mathcal{F}$ with the larger domain $\mathcal{S}(2.28)$ as discussed in [5]. After performing the modulus integral explicitly, we find the relation (3.1) is really satisfied.

Let us finally argue on the decompactification limit $\lim _{R_{-} \rightarrow \infty} \frac{Z_{\text {torus }}(\beta)}{\sqrt{2} \pi R_{-}}$as we promised. We can again show that only the $W=0$ sector survives in this limit. The summations with respect to $p, q$ reduce to integrals and easily carried out. We hence obtain

$$
\begin{aligned}
\lim _{R_{-} \rightarrow \infty} \frac{Z_{\text {torus }}(\beta)}{\sqrt{2} \pi R_{-}}=2 \pi R_{T} & \frac{\beta}{4 \pi^{2} \alpha^{\prime}} \int_{\mathcal{F}} \frac{d^{2} \tau}{\tau_{2}^{2}} \sum_{\epsilon_{i}=0,1} \sum_{\substack{w \in 2 \mathbf{Z}_{+\epsilon_{1}} \\
n \in 2 \mathbf{Z}+\epsilon_{2}}} e^{-\frac{\beta^{2}|w \tau-n|^{2}}{4 \pi \alpha^{\prime} \tau_{2}}} \\
& \times F_{\epsilon_{1}, \epsilon_{2}}\left(\tau, \bar{\tau} ;-i \frac{\sqrt{2} \mu \beta w}{4 \pi}, i \frac{\sqrt{2} \mu \beta n}{4 \pi}, \frac{\sqrt{2} \mu \beta}{4 \pi \tau_{2}}|w \tau-n|\right),
\end{aligned}
$$

where we set

$$
F_{\epsilon_{1}, \epsilon_{2}}(\tau, \bar{\tau} ; \alpha, \beta, m)=\frac{\Theta_{\left(\frac{1}{2} \alpha+\frac{1}{2} \epsilon_{1}, \frac{1}{2} \beta+\frac{1}{2} \epsilon_{2}\right)}(\tau, \bar{\tau} ; m)^{4}}{\Theta_{(0,0)}(\tau, \bar{\tau} ; m)^{3} \cdot \Theta_{(\alpha, \beta)}(\tau, \bar{\tau} ; m)} .
$$

The modular invariance in (3.30) is similarly checked. Moreover, we can again rewrite it by setting $w=0$ and replacing the integration region $\mathcal{F}$ with $\mathcal{S}$. Transforming the integration variable as $\tau=\tau_{1}+i \frac{\sqrt{2} \beta n}{4 \pi \alpha^{\prime} p^{+}}$, we can again show the relation (3.1) with (2.31). 


\section{Comments}

In this paper we have calculated the thermal partition function (free energy) of superstring on the compactified pp-wave. We have presented two analyses, that is, the operator formalism and path-integral approach. We have also checked their equivalence. Now, the several comments are in order:

1. We have observed that the winding modes along the transverse circle non-trivially depend on the longitudinal quantum numbers in the DLCQ model, and are decoupled in the nonDLCQ model. These aspects are in a sharp contrast with the usual toroidal compactifications in flat backgrounds. We here illustrate why such peculiar phenomenon occurs:

We first point out that it depends on the modulus $\tau$ whether the string can wind around the space-like circle contrary to the flat cases. This is essentially due to the fact that we are working on the time-dependent frame. In the DLCQ model we effectively have the discretized moduli space, and we observed that the winding modes consistently exist at each point of the discretized modulus.

On the other hand, we have the continuous modulus which should be integrated out in the non-DLCQ model. However, the existence of winding modes limits the modulus to "rational points", which have the zero measure and do not contribute to the modulus integral. In other words, recall that the physical spectrum does not really degenerate with respect to the canonical momentum $k$ unless $W=0$. Although the momentum $k$ does not appear in the Hamiltonian (2.14), it controls the physical spectrum through the level matching condition (2.15). Only in the case $W=0$ we have the infinite degeneracy of Landau levels which cancels the infinitely small factor $1 / \mathcal{N}$.

2. The analysis on the Hagedorn behavior [24] is quite easy, since we have already known the modular invariant forms (3.29), (3.30). We follow the argument given in [15]. Namely, it is enough to investigate the IR behavior of the term with $w=1, n=W=0$ in (3.29) (or (3.30)), which could be tachyonic at a sufficiently high temperature and brings about the thermal instability as in the flat backgrounds $[6,7]$. We can show that

$$
Z_{\text {torus }}(\beta): \text { finite } \Longleftrightarrow \beta>\beta_{H}
$$

with the equation determining the Hagedorn temperature $T_{H} \equiv \beta_{H}^{-1}$;

$$
\frac{\beta_{H}^{2}}{8 \pi^{2} \alpha^{\prime}}=8 \Delta\left(\frac{\sqrt{2} \mu \beta_{H}}{4 \pi} ;-i \frac{\sqrt{2} \mu \beta_{H}}{8 \pi}+\frac{1}{2}\right)-6 \Delta\left(\frac{\sqrt{2} \mu \beta_{H}}{4 \pi} ; 0\right)-2 \Delta\left(\frac{\sqrt{2} \mu \beta_{H}}{4 \pi} ;-i \frac{\sqrt{2} \mu \beta_{H}}{4 \pi}\right)
$$


where the zero-point energy $\Delta(m ; a)$ of the massive theta functions is defined in (A.2). The equation (4.2) does not depend on the DLCQ radius $R_{-}$as well as the compactification radius $R_{T}$, as should be (see [22]). We should here note that the Hagedorn temperature determined by (4.2) is not equal to that of the original coordinate system in $[2,3,4]$, which is studied in $[13,14,15,20]$, even under the uncompactified model $R_{T}=\infty$. One might suppose this fact peculiar, but it is not a contradiction. The temperature is defined associated to the Euclidean time axis, and hence should be a quantity not independent of the choice of space-time coordinates.

On the other hand, we know a manifestly coordinate free quantity; the cosmological constant. So, one might ask how we can avoid the contradiction even though the partition function (3.23) (or (3.29)) apparently has a different form compared with that calculated in the original coordinate [15]. To answer this question, we first note that the vacuum energy densities should be compared at the zero-temperature limit $\beta \rightarrow \infty$, because the temperature is not coordinate free as pointed out above. Moreover, the amplitude in the zero-temperature limit is described by the sectors with no thermal winding $w=n=0$. We thus have to confirm that the contribution from this sector, which we omitted in our analyses, does not depend on the choice of coordinate system. In particular, we must show that it really vanishes, as is expected from the space-time SUSY. In a naive sense this is very easy to confirm. In fact, in our instanton gauge we have $Z^{+}=$const., if $w=n=0$ holds, leading to the 8 massless bosons and 8 massless Dirac fermions in the transverse sector as in the flat backgrounds. We hence trivially obtain the vanishing cosmological constant irrespective of the choice of coordinate system. However, the gauge choice $Z^{+}=$const. is supposed to be the "singular point" of the light-cone gauge $p^{+}=0$, which is not compatible with the usual Virasoro constraints. We thus may need more careful study about this problem, probably with introducing a suitable regularization scheme to evaluate the vacuum energy ${ }^{2}$.

3. Generalizations to the compactifications on higher dimensional space-like tori are straightforward. Among others, let us consider the $T^{2}$-compactification in which a circle lies along the $X^{1}-X^{2}$ plane and another circle does along the $X^{3}-X^{4}$ plane, for example. In that case we should work on the rotated frame generalizing (2.1). We find 20 Killing spinors [8]

\footnotetext{
${ }^{2} \mathrm{~A}$ possible way to evaluate it may be to first calculate it with the small non-zero $p^{+}$, and then to take the $p^{+} \rightarrow 0$ limit. With the non-zero $p^{+}$the partition function could not vanish. However, since we are now considering the vacuum energy density, the result actually seems to vanish because of the infinite volume factor due to the non-compact background. (See [15] for the more detail.)
} 
and obtain the same spectra of the bosonic and fermionic oscillators as follows;

$$
2 \times\left(\omega_{n}+m\right), \quad 2 \times\left(\omega_{n}-m\right), \quad 4 \times \omega_{n}, \quad\left(n \in \mathbf{Z}, \omega_{n} \equiv \sqrt{n^{2}+m^{2}}\right) .
$$

This fact suggests that some of Killing spinors are "time-independent" and define the supercharges commuting with the world-sheet Hamiltonian. The similar analysis gives the following thermal partition function (in the DLCQ model);

$$
\begin{aligned}
& Z_{\text {torus }}(\beta)=\prod_{i=1,2}\left(2 \pi R_{T}^{i}\right) \nu \int_{\mathcal{F}} \frac{d^{2} \tau}{\tau_{2}^{2}} \sum_{\epsilon_{i}=0,1} \sum_{\substack{w \in 2 \mathbf{Z}_{+\epsilon_{1}} \\
n \in 2 \mathbf{Z}+\epsilon_{2}}}^{\prime} \sum_{W^{i} \in(p / d) \mathbf{Z}} e^{-\frac{\beta^{2}|w \tau-n|^{2}}{4 \pi \alpha^{\prime} \tau_{2}}\left(1+\frac{R_{-}^{2} \sum_{i}\left(R_{T}^{i} W^{i}\right)^{2}}{2 p^{2} \alpha^{\prime 2}}\right)} \\
& \times \tau_{2} \delta^{(2)}((w \nu+i p) \tau-(n \nu+i q))
\end{aligned}
$$

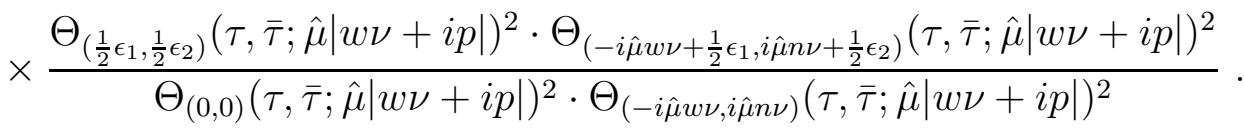

$$
\begin{aligned}
& =\prod_{i=1,2}\left(2 \pi R_{T}^{i}\right) \sum_{p, q} \sum_{W^{i} \in(p / d) \mathbf{Z}}\left[\sum_{n: \text { even }, n>0} \frac{1}{n p} e^{-\frac{\beta^{2} n^{2}}{4 \pi \alpha^{\prime} \tau_{2}}\left(1+\frac{R_{-}^{2} \sum_{i}\left(R_{T}^{i} W^{i}\right)^{2}}{2 p^{2} \alpha^{\prime 2}}\right)}\right. \\
& \left.+\sum_{n: \text { odd }, n>0} \frac{1}{n p} e^{-\frac{\beta^{2} n^{2}}{4 \pi \alpha^{\prime} \tau_{2}}\left(1+\frac{R_{-}^{2} \sum_{i}\left(R_{T}^{i} W^{i}\right)^{2}}{2 p^{2} \alpha^{\prime 2}}\right)} \frac{\Theta_{\left(0, \frac{1}{2}\right)}(\tau, \bar{\tau} ; \hat{\mu} p)^{2} \cdot \Theta_{\left(0, i \hat{\mu} n \nu+\frac{1}{2}\right)}(\tau, \bar{\tau} ; \hat{\mu} p)^{2}}{\Theta_{(0,0)}(\tau, \bar{\tau} ; \hat{\mu} p)^{2} \cdot \Theta_{(0, i \hat{\mu} n \nu)}(\tau, \bar{\tau} ; \hat{\mu} p)^{2}}\right]
\end{aligned}
$$

where $R_{T}^{1}, R_{T}^{2}$ are the compactification radii. We again set $\tau \equiv \frac{q+i n \nu}{p}$ in the second line and the summations of $p, q$ and $n$ are taken in the range such that $\tau \in \mathcal{S}$. These are quite reminiscent forms of those given in [15]. The first line is the modular invariant expression and the second line only contains the contributions from physical states. The first term in the second line (the terms with even $n$ ) corresponds to the Witten index and the second one describes the thermal excitations.

4. We have various future directions for this work. A natural generalization of our calculations is to include the non-vanishing chemical potential as in $[14,20]$. As for the gauge theory duals, the DLCQ model for the uncompactified pp-wave was studied in [25, 26], and more recently the compactified models with/without DLCQ have been investigated in [23]. It may be interesting to compare the thermodynamical aspects in these dual field theories with our string calculations. Moreover, it will be a tractable and interesting problem to analyze the compactified pp-wave backgrounds originating from the $A d S_{3} \times S^{3}$ backgrounds, and to discuss the aspects of holographic dualities as in [27]. Among other things, it is well-known that we have the $S L(2 ; \mathbf{Z})$-family of supersymmetric vacua possessing both the RR and NSNS flux in those cases. There would appear non-trivial zero-modes which could allow new winding 
sectors under the suitable choice of flux. We would like to explore this aspect in a detail elsewhere.

\section{Acknowledgement}

I would like to thank Y. Imamura, T. Kawano, S. Mizoguchi, T. Takayanagi and S. Yamaguchi for stimulating conversations. This work is supported in part by a Grant-inAid for the Encouragement of Young Scientists ( $¥ 13740144)$ from the Japanese Ministry of Education, Culture, Sports, Science and Technology. 


\section{Appendix A : Massive Theta Functions}

The "massive theta functions" are defined as

$$
\Theta_{(a, b)}(\tau, \bar{\tau} ; m) \stackrel{\text { def }}{=} e^{4 \pi \tau_{2} \Delta(m ; a)} \prod_{n \in \mathbf{Z}}\left|1-e^{-2 \pi \tau_{2} \sqrt{m^{2}+(n+a)^{2}}+2 \pi i \tau_{1}(n+a)+2 \pi i b}\right|^{2},
$$

where the regularized zero-point energy $\Delta(m ; a)$ is defined $[10,11,14,15]$ as

$$
\begin{aligned}
\Delta(m ; a) & \stackrel{\text { def }}{=} \frac{1}{2} \sum_{n \in \mathbf{Z}} \sqrt{m^{2}+(n+a)^{2}}-\frac{1}{2} \int_{-\infty}^{\infty} d k \sqrt{m^{2}+k^{2}} \\
& =-\frac{1}{2 \pi^{2}} \sum_{n=1}^{\infty} \int_{0}^{\infty} d s e^{-s n^{2}-\frac{\pi^{2} m^{2}}{s}} \cos (2 \pi n a) .
\end{aligned}
$$

They have the following modular properties [11]

$$
\begin{aligned}
& \Theta_{(a, b)}(\tau+1, \bar{\tau}+1 ; m)=\Theta_{(a, b+a)}(\tau, \bar{\tau} ; m) \\
& \Theta_{(a, b)}(-1 / \tau,-1 / \bar{\tau} ; m|\tau|)=\Theta_{(b,-a)}(\tau, \bar{\tau} ; m) .
\end{aligned}
$$

The next formulas are also useful;

$$
\begin{aligned}
& \Theta_{(a, b)}(\tau, \bar{\tau} ; m)=\Theta_{(-a,-b)}(\tau, \bar{\tau} ; m)=\Theta_{(a+r, b+s)}(\tau, \bar{\tau} ; m), \quad\left({ }^{\forall} r, s \in \mathbf{Z}\right), \\
& \lim _{m \rightarrow 0} \Theta_{(a, b)}(\tau, \bar{\tau} ; m)=e^{-2 \pi \tau_{2} a^{2}}\left|\frac{\theta_{1}(\tau, a \tau+b)}{\eta(\tau)}\right|^{2} .
\end{aligned}
$$

The partition function for the $d$-components complex massive boson (non-chiral fermion) with the boundary conditions $\phi(z+2 \pi, \bar{z}+2 \pi)=e^{2 \pi i a} \phi(z, \bar{z}), \phi(z+2 \pi \tau, \bar{z}+2 \pi \bar{\tau})=e^{-2 \pi i b} \phi(z, \bar{z})$, is calculated as

$$
\begin{aligned}
Z(\tau, \bar{\tau} ; m) & =\operatorname{Tr}\left[(-1)^{\mathbf{F}} e^{-2 \pi \tau_{2} H+2 \pi i \tau_{1} \hat{P}+2 \pi i b \hat{h}}\right] \\
& =\Theta_{(a, b)}(\tau, \bar{\tau} ; m)^{-\epsilon d}
\end{aligned}
$$

where $\epsilon=+1$ for the boson and $\epsilon=-1$ for the fermion. In this expression we introduced the momentum operator for the twisted fields

$$
\hat{P}=\sum_{n}\left((n+a) N_{n}^{(+)}+(n-a) N_{n}^{(-)}\right)
$$

and the "helicity operator"

$$
\hat{h}=\sum_{n}\left(N_{n}^{(+)}-N_{n}^{(-)}\right)
$$

where $N_{n}^{(+)}, N_{n}^{(-)}$express the mode counting operators associated to the Fourier modes $e^{ \pm i(n+a) \sigma}, e^{ \pm i(n-a) \sigma}$ respectively. 


\section{References}

[1] R. Güven, Phys. Lett. B 191, 275 (1987); D. Amati and C. Klimcik, Phys. Lett. B 210, 92 (1988); G. T. Horowitz and A. R. Steif, Phys. Rev. Lett. 64, 260 (1990); G. T. Horowitz and A. R. Steif, Phys. Rev. D 42, 1950 (1990); A. R. Steif, Phys. Rev. D 42, 2150 (1990); A. A. Tseytlin, Phys. Lett. B 288, 279 (1992) [arXiv:hep-th/9205058]; A. A. Tseytlin, Nucl. Phys. B 390, 153 (1993) [arXiv:hep-th/9209023]; Phys. Rev. D 47, 3421 (1993) [arXiv:hep-th/9211061]; E. A. Bergshoeff, R. Kallosh and T. Ortin, Phys. Rev. D 47, 5444 (1993) [arXiv:hep-th/9212030].

[2] M. Blau, J. Figueroa-O'Farrill, C. Hull and G. Papadopoulos, JHEP 0201 (2002) 047, hep-th/0110242; Class. Quant. Grav. 19 (2002) L87, hep-th/0201081.

[3] R. R. Metsaev, Nucl. Phys. B 625 (2002) 70, hep-th/0112044; R. R. Metsaev and A. A. Tseytlin, Phys. Rev. D 65, 126004 (2002) [arXiv:hep-th/0202109].

[4] D. Berenstein, J. M. Maldacena and H. Nastase, JHEP 0204 (2002) 013, hep-th/0202021.

[5] J. Polchinski, Commun. Math. Phys. 104, 37 (1986).

[6] B. Sathiapalan, Phys. Rev. D 35, 3277 (1987); Y. I. Kogan, JETP Lett. 45, 709 (1987) [Pisma Zh. Eksp. Teor. Fiz. 45, 556 (1987)]; K. H. O'Brien and C. I. Tan, Phys. Rev. D 36, 1184 (1987).

[7] J. J. Atick and E. Witten, Nucl. Phys. B 310, 291 (1988).

[8] J. Michelson, Phys. Rev. D 66, 066002 (2002) [arXiv:hep-th/0203140].

[9] J. G. Russo and A. A. Tseytlin, JHEP 0204 (2002) 021, hep-th/0202179.

[10] O. Bergman, M. R. Gaberdiel and M. B. Green, arXiv:hep-th/0205183.

[11] T. Takayanagi, arXiv:hep-th/0206010.

[12] Y. Hikida and Y. Sugawara, JHEP 0210, 067 (2002) [arXiv:hep-th/0207124].

[13] L. A. Pando Zayas and D. Vaman, arXiv:hep-th/0208066.

[14] B. R. Greene, K. Schalm and G. Shiu, arXiv:hep-th/0208163.

[15] Y. Sugawara, Nucl. Phys. B 650 (2003) 75, [arXiv:hep-th/0209145].

[16] A. Sinha and N. V. Suryanarayana, JHEP 0211, 026 (2002) [arXiv:hep-th/0209247]. 
[17] A. B. Hammou, JHEP 0211, 028 (2002) [arXiv:hep-th/0209265].

[18] J. F. Morales, arXiv:hep-th/0210229.

[19] M. R. Gaberdiel and M. B. Green, arXiv:hep-th/0211122.

[20] R. C. Brower, D. A. Lowe and C. I. Tan, arXiv:hep-th/0211201.

[21] L. Susskind, hep-th/9704080; A. Sen, hep-th/9709220; N. Seiberg, Phys. Rev. Lett. 79 (1997) 3577, hep-th/9710009.

[22] G. Grignani and G. W. Semenoff, Nucl. Phys. B 561, 243 (1999) [arXiv:hep-th/9903246];

G. Grignani, P. Orland, L. D. Paniak and G. W. Semenoff, Phys. Rev. Lett. 85, 3343 (2000) [arXiv:hep-th/0004194]; G. W. Semenoff, arXiv:hep-th/0009011; Nucl. Phys. Proc. Suppl. 108, 99 (2002) [arXiv:hep-th/0112043].

[23] M. Bertolini, J. de Boer, T. Harmark, E. Imeroni and N. A. Obers, arXiv:hep-th/0209201.

[24] R. Hagedorn, Nuovo Cim. Suppl. 3 (1965) 147.

[25] S. Mukhi, M. Rangamani and E. Verlinde, JHEP 0205, 023 (2002) [arXiv:hepth/0204147].

[26] M. Alishahiha and M. M. Sheikh-Jabbari, Phys. Lett. B 538, 180 (2002) [arXiv:hepth/0204174].

[27] A. Parnachev and D. A. Sahakyan, JHEP 0206, 035 (2002) [arXiv:hep-th/0205015]; Y. Hikida and Y. Sugawara, JHEP 0206, 037 (2002) [arXiv:hep-th/0205200]; S. R. Das and C. Gomez, JHEP 0207, 016 (2002) [arXiv:hep-th/0206062]; O. Lunin and S. D. Mathur, Nucl. Phys. B 642, 91 (2002) [arXiv:hep-th/0206107]; J. Gomis, L. Motl and A. Strominger, JHEP 0211, 016 (2002) [arXiv:hep-th/0206166]; E. Gava and K. S. Narain, JHEP 0212, 023 (2002) [arXiv:hep-th/0208081]. 